\title{
Pentaquarks: the latest experimental results
}

\author{
M.Battaglieri*, R. De Vita*, V.Kubarovsky ${ }^{\dagger, * *}$ and the CLAS Collaboration \\ ${ }^{*}$ Istituto Nazionale di Fisica Nucleare - Via Dodecaneso 3316139 Genova ITALY \\ ${ }^{\dagger}$ Rensselaer Polytechnic Institute - Troy New York 12180-359 \\ ** Jefferson Laboratory - 12000 Jefferson Avenue Newport News 23606 Virginia
}

\begin{abstract}
After the claim of the possible discovery of a pentaquark state, many experiments reported positive and negative results opening a discussion about the pentaquark existence. New experiments with high resolution and high statistics are needed in the reaction channels and for the kinematics of the positive results to solve the controversy. Jefferson Lab started a comprehensive program to search for pentaquark in photoproduction at threshold on proton and deuteron targets, collecting more than 10 times the existing statistics. The first experiment on the proton $(g 11)$ just finished to analyze the data, and the first results of the pentaquark search are reported here.
\end{abstract}

Keywords: Pentaquark, photoproduction, proton target.

PACS: $13.60 \mathrm{Rj} ; 25.20 \mathrm{Lj}$

\section{INTRODUCTION}

All known hadronic matter is composed of two kinds of quark configurations: baryons, such as protons and neutrons, which are combinations of 3 quarks (qqq), and mesons, such as pions, which are combinations of a quark and an anti-quark $(q \bar{q})$. However, states of matter consisting of four quarks and an anti-quark $(q q q q \bar{q})$, called pentaquarks, are expected by the theory of Quantum Chromodynamics. In particular, states where the anti-quark has a different flavor with respect to the others have quantum numbers that are not allowed for standard particles and clearly reveal their 'exotic' nature. In the past, experimental searches focused on the search for pentaquark states, did not provide any clear evidence, leading to the conclusion that discoveries in this field would not be possible in the short term. As a consequence, this particular experimental program was abandoned and no further data analysis was pursued for many years. However, the lack of experimental proof left an open question about the physical manifestation of lowenergy QCD. In spite of that, theoretical interest in this field has continued and, recently, Diakonov and collaborators [1] made definite predictions about the masses and widths of a decuplet of pentaquark states (the so-called "antidecuplet"). The most intriguing aspect of such a multiplet is the presence of three states with exotic quantum numbers: the $\Theta^{+}, S=+1$, and the $\Xi^{--}$and $\Xi^{+}$with $S=-2$. In particular the $\Theta^{+}$strangeness, never observed in the baryon sector and not compatible with a $q q q$ state, requires at least a pentaquark configuration of the type $u u d d \bar{s}$. The mass of the $\Theta^{+}$was predicted to be quite low $(1540 \mathrm{MeV})$ and its widths very narrow ( 10-15 MeV) implying that if such state exists it should be directly visible in the measured invariant masses without need for more sophisticated Partial Wave Analysis. 


\section{THE POSITIVE RESULTS}

The first evidence of the $\Theta^{+}$was obtained in the photoproduction measurement performed by the LEPS Collaboration [2]. The signal was found in the reaction $\gamma n \rightarrow$ $K^{-} \Theta^{+} \rightarrow K^{-} K^{+} n$. The target was a $\mathrm{CH}_{2}$ scintillator located just downstream of the primary hydrogen $\left(\mathrm{LH}_{2}\right)$ target. The signal was seen in the missing mass spectrum of the $K^{-}$, after cutting out sources of background from $\phi$ and $\Lambda(1520)$ production. Corrections to compensate for the neutron's Fermi motion in the carbon nucleus were applied. The final peak was found at a mass of $1540 \pm 10 \mathrm{MeV}$, with a width less than $25 \mathrm{MeV}$, and a gaussian significance of $4.6 \sigma$. This result was shortly thereafter confirmed by the CLAS Collaboration [3] who analyzed existing data on deuteron target looking at the channel $\gamma d \rightarrow K^{-} K^{+} n p$. Events with the $K^{-} K^{+} p$ in the final state were selected and the missing mass technique was used to identify the neutron. A peak was observed in the $n K^{+}$invariant mass at a value of $1542 \pm 5 \mathrm{MeV}$, with a width less than $21 \mathrm{MeV}$, and a statistical significance of more than $5 \sigma$. Evidence of a $\Theta^{+}$candidate was also found in the reaction $\gamma p \rightarrow K^{-} \pi^{+} \Theta^{+} \rightarrow K^{-} \pi^{+} K^{+} n$ [4]. DIANA Collaboration re-analyzed the old $K$-Xenon bubble-chamber data [5] $\left(K^{+} n \rightarrow\left(\bar{K}^{0}\right) \Theta^{+} \rightarrow\left(\bar{K}^{0}\right) \pi^{+} \pi^{-} p\right)$ and reported a narrow peak $(\sim 10 \mathrm{MeV})$, located at $1.54 \mathrm{GeV}$ with a statistical significance of $4 \sigma$. The SAPHIR Collaboration reported about the reaction $\gamma p \rightarrow \bar{K}^{0} \Theta^{+} \rightarrow K_{S} K^{+}(n)$ where a $5 \sigma$ peak was isolated in the $K_{S}$ missing mass. The peak was found to be at $1540 \pm 5$ $\mathrm{MeV}$ and a large production cross section of $300 \mathrm{nb}$ was quoted in the paper [6]. Evidence of a narrow structure in the $\left(K_{S} p\right)$ system was also reported in neutrino and anti-neutrino collisions with nuclei [7] at CERN. A peak of less than $20 \mathrm{MeV}$ width resonance was found at a mass of $1533 \pm 5 \mathrm{MeV}$ with a statistical significance of $6.7 \sigma$. A narrow $4 \sigma$ peak in the same invariant mass spectrum, located at $1526 \pm 3 \mathrm{MeV}$, was also found by the Hermes Collaboration [8] in the analysis of quasi-real photo production on a deuteron target. Another positive evidence was also found in $p p$ scattering at few GeV beam energy (COSY-TOF Collaboration [9]). Positive results were then reported analyzing collisions of high energy proton beams against electron beam (ZEUS Collaboration [10]) and fixed targets (SVD-2 Collaborations [11]).

Are all these evidences enough to prove that the pentaquark does exist? The possible discovery of a state beyond ordinary matter triggered the interest of the scientific community, resulting in the publication of more than 500 related papers in the past two years.

\section{THE NEGATIVE RESULTS}

First doubts on the pentaquark existence, arise from the fact that, the mass of the $\Theta^{+}$ measured in the different experiments were not compatible within the quoted errors. In particular, there is a systematic difference for the mass value found in the two possible decay modes: reported values were lower for $\Theta^{+} \rightarrow p K^{0}$ than for $\Theta^{+} \rightarrow n K^{+}$. The difference in the background or interference effects that systematically affect the two decay modes in a different way could explain the difference. One can simply assume that the quoted errors on the measured masses were underestimated but a possible indication of a serious concern about the $\Theta^{+}$existence should not be ignored. 
In the past year reanalysis of data collected in high energy experiments $[12,13,14$, $15,16,17,18,19,20,21,22,23,24,25]$ show no evidence for pentaquarks. Data were collected in different laboratories (SLAC, DESY, CERN, FERMILAB, BNL) using a variety of different probes $\left(e^{+} e^{-}\right.$colliders, heavy-ion and high-energy proton-proton and proton-nuclei interactions) and, for the most part of the results, analyzing a huge statistics.

Was it enough to prove that the pentaquark did not exist? The answer is not so simple. As an example we report the argument to exclude the significance of the $e^{+} e^{-}$ experiments. It is well known that hadroproduction abundance in $e^{+} e^{-}$collisions as a function of the produced hadron mass has a very steep slope that depends on the number of quark-pairs that have to be generated from the vacuum. Pseudo-scalar mesons (one $q \bar{q}$ pair) are produced with a slope of $\sim 10^{-2} / \mathrm{GeV}$ while baryons (two $q \bar{q}$ pairs more) have $\sim 10^{-4} / \mathrm{GeV}$. Following this logic, one can predict that pentaquarks (four $q \bar{q}$ pairs more) should have a slope of $\sim 10^{-8} / \mathrm{GeV}$ that makes the huge statistics collected by $e^{+} e^{-}$colliders, useless to draw any conclusion.

\section{SECOND GENERATION EXPERIMENTS}

In summary, twelve different laboratories, using different probes (photons, electrons, protons, neutrinos) and targets (protons, neutrons, nuclei) reported positive results. Some of them, with a high statistical significance. Limiting aspects of the so-called 'low energy' experiments were: the most part of the observed structures contained only few counts, all peaks did not show at the same mass, the background shape, necessary to define a possible signal over it, was not theoretically understood and the limited statistics prevented to derive a smooth curve directly from the data. Moreover many analyses used strong kinematic cuts to enhance the signal-to-background ratio that may distort the spectra and some experiment did not tag the strangeness of the final state.

Ten 'high energy' experiments reported null results analyzing high statistics data sets deriving stringent upper limits on the $\Theta^{+}$production but the different kinematic conditions, which likely involved dissimilar production mechanisms, the unknown relative weight of background reactions that could blind the small pentaquark production at high energies, and the different acceptance of the different detectors make very difficult direct comparisons of the results of the different experiments, preventing a definitive conclusion about the pentaquark's existence.

A second generation of dedicated experiments, optimized for the pentaquark search, was undertaken at Jefferson Lab. These experiments cover the few $\mathrm{GeV}$ beam energy region where most of the positive evidence were reported, with each collecting at least an order of magnitude more statistics than any of the previous measurements. The mass resolutions are approximately a few $\mathrm{MeV}$ and the accuracy of the mass determination is approximately 1-2 MeV, allowing precise determination of any possible narrow peaks in the decay distributions. All used real photon in different energy ranges and different targets. Two experiments ( $g 10$ and $e g 3$ ) were in the range $1.0-3.0 \mathrm{GeV}$ and $4.0-5.4 \mathrm{GeV}$ respectively and used a liquid deuteron target while one experiment $(g 11)$ used a proton target with a photon beam of 1.6-3.8 GeV. The three experiments collected data and now they are completing the physics analyses. 

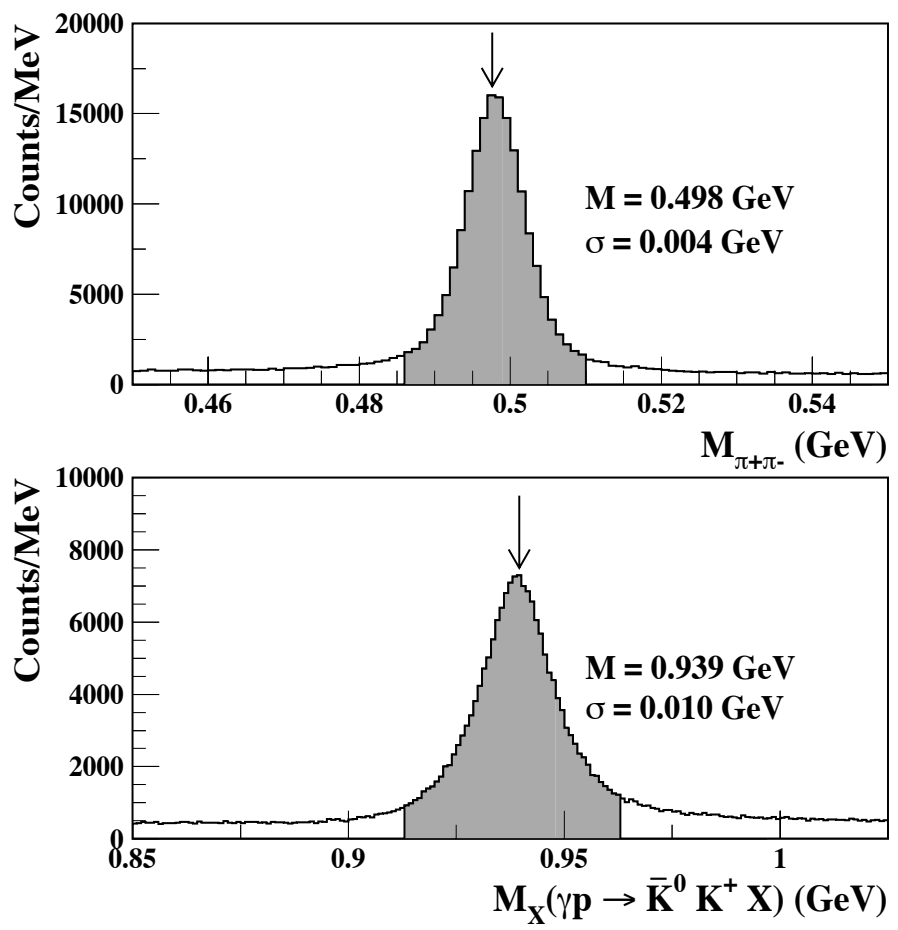

FIGURE 1. Bottom: missing mass for the reaction $\gamma p \rightarrow \bar{K}^{0} K^{+} X$ after $\bar{K}^{0}$ selection showing a peak at the neutron mass. The mass positions and widths of the measured peaks are given. For comparison, the arrows indicate the accepted value for the mass position. The shaded area corresponds to the events used in the analysis.

\section{THE G11 EXPERIMENT AT JLAB}

Here we present the first result of of the $g 11$ experiment reporting the result of the exclusive measurement of the reaction $\gamma p \rightarrow \bar{K}^{0} K^{+} n$. The reconstruction of all participating particles (exclusive measurement) allows one to tag the strangeness of the reaction which clearly identifies the exotic nature of the baryon produced in association with the $\bar{K}_{0}$. This channel was previously investigated at ELSA by the SAPHIR collaboration in a similar photon energy range, finding positive evidence for a narrow $\Theta^{+}$state with $\mathrm{M}=1540 \mathrm{MeV}$ and full width half maximum (FWHM) $\Gamma<25 \mathrm{MeV}$. They originally quoted a total production cross section of the order of $300 \mathrm{nb}$ (later reduced to 50 $\mathrm{nb}$ [26]). For the first time, the new results put previous findings to a direct test.

The measurement was performed using the CLAS detector in Hall B with a bremsstrahlung photon beam produced by a continuous $60 \mathrm{nA}$ electron beam of $E_{0}=$ $4.0 \mathrm{GeV}$ impinging on a gold foil $8 \times 10^{-5}$ radiation lengths thick. A bremsstrahlung tagging system with a photon energy resolution of $0.1 \% E_{0}$ was used to tag photons in the energy range from $1.6-3.8 \mathrm{GeV}$. A liquid hydrogen target was contained in a mylar cylinder cell $4 \mathrm{~cm}$ in diameter and $40 \mathrm{~cm}$ long. Outgoing hadrons were detected in the 


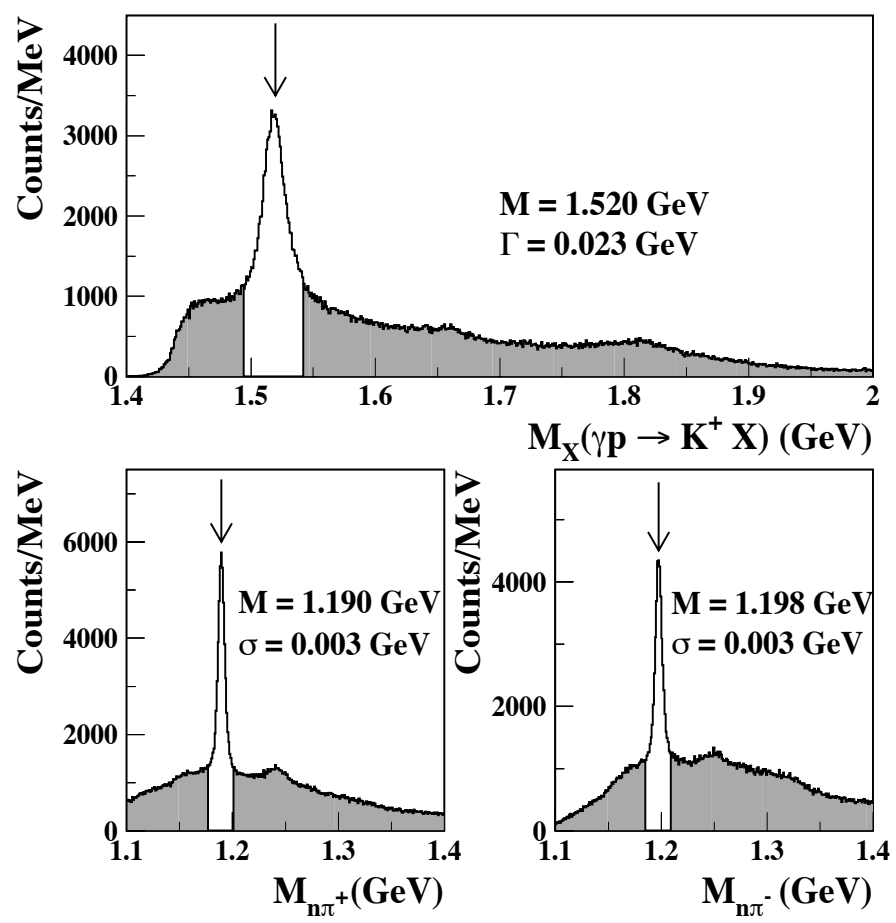

FIGURE 2. Top: $K^{+}$missing mass distribution with the $\Lambda^{*}(1520)$ peak. Bottom: $n \pi^{+}$(left) and $n \pi^{-}$ (right) invariant mass distributions with $\Sigma^{+}(1189)$ and $\Sigma^{-}(1197)$ peaks. The mass position and width of the measured peaks are indicated. For comparison, the arrows indicate the accepted value for the mass position. The shaded area corresponds to the events used in the analysis.

CLAS [27] spectrometer. Momentum information for charged particles was obtained via tracking through three regions of multi-wire drift chambers immersed in a toroidal magnetic field $(\sim 0.5 \mathrm{~T})$, which was generated by six superconducting coils. The field was set to bend the positive particles away from the beam into the acceptance region of the detector. Time-of-flight scintillators (TOF) were used for hadron identification. The interaction time between the incoming photon and the target was measured by the Start Counter (ST), consisting of a set of 24, $2.2 \mathrm{~mm}$ thick plastic scintillators surrounding the hydrogen cell. The CLAS momentum resolution is of the order of $0.5-1 \%$ depending on the kinematics. The detector geometrical acceptance for each positive particle in the relevant kinematic region is about $40 \%$. It is somewhat less for low energy negative hadrons, which can be lost at forward angles because they are bent out of the acceptance by the toroidal field. Coincidences between the photon tagger and the CLAS detector triggered the recording of the events. The trigger in CLAS was defined requiring the coincidence between the TOF system and the ST in at least two sectors. We took data for 50 days during June and July 2004 collecting more than 7G triggers corresponding to an integrated luminosity of $70 \mathrm{pb}^{-1}$. This is probably the highest statistics ever collected in experiments with tagged photons. 


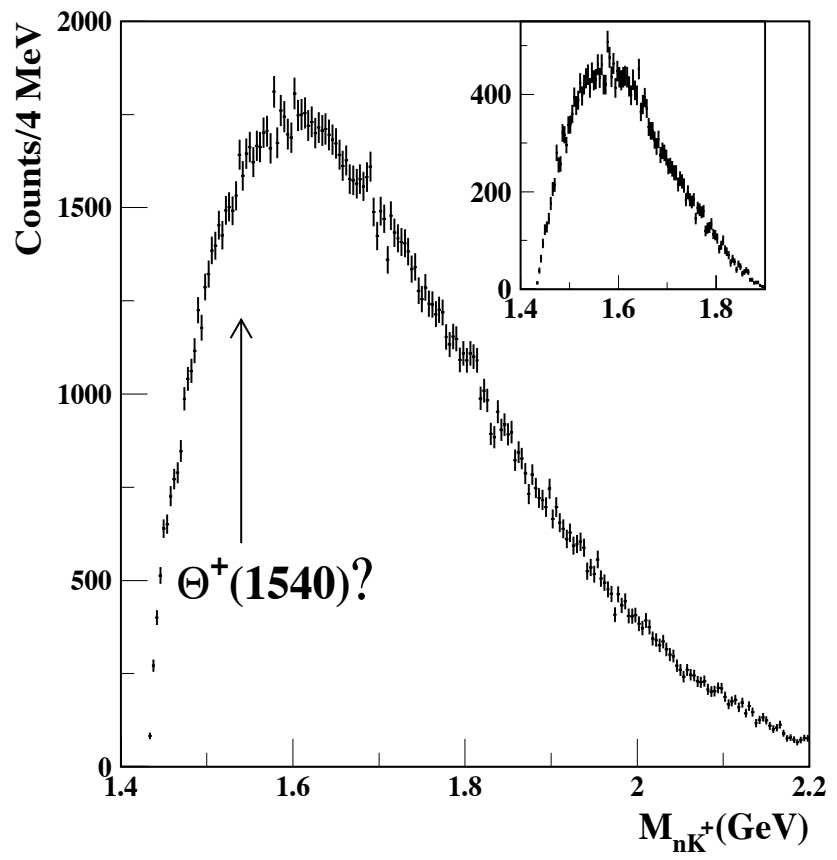

FIGURE 3. The $n K^{+}$invariant mass distribution after all cuts. It is smooth and no narrow structures are evident. The arrow shows the position where evidence for the $\Theta^{+}$was found by previous experiments. The inset shows the $n K^{+}$mass distribution with specific cuts to reproduce the SAPHIR analysis [6] as described in the text.

\section{FIRST RESULTS}

The reaction $\gamma p \rightarrow K^{-} \Theta^{+} \rightarrow \bar{K}^{0} K^{+} n$ was isolated as follows. The $K^{+}$was detected directly in the magnetic spectrometer, and the $K^{0}$ was reconstructed from its $\pi^{+} \pi^{-}$ decay. The momentum and energy of the neutron were reconstructed from the known incident photon energy and measurements of all other particles in the event. The quality of the channel identification is shown in Figure 1 where the $K_{S}^{0}$ and the missing neutron peaks are seen above a small background. Reactions involving decay of hyperons, contribute to the same final state. The most sizable are: $\gamma p \rightarrow K^{+} \Lambda^{*}(1520) \rightarrow K^{+} \bar{K}^{0} n$, $\gamma p \rightarrow \pi^{-} K^{+} \Sigma^{+}$, and $\gamma p \rightarrow \pi^{+} K^{+} \Sigma^{-}$. Figure 2 shows the background hyperon peaks: $\Lambda^{*}(1520)$ in the $K^{+}$missing mass spectrum and the $\Sigma^{+}, \Sigma^{-}$peaks in the $n \pi^{+}$and $n \pi^{-}$ invariant mass spectra respectively. The mass region of each of these hyperon peaks was excluded from the final data set, as shown from the shaded regions in the same figure. While they represent a background to the pentaquark search, but easily removable in our analysis with a cut around their masses, they can be used as a check of the entire procedure, e.g. by extracting their production cross sections and comparing to the world data. After all cuts, the data sample contains approximately $0.17 \times 10^{6}$ events out of the 
$7 \times 10^{9}$ in the original data set. The resulting $n K^{+}$invariant mass distribution is shown in Fig. 3. The distribution is smooth and structureless. In particular, no evidence for a peak or an enhancement is observed at masses near $1540 \mathrm{MeV}$, where signals associated with the $\Theta^{+}$were previously reported. To enhance a possible resonance signal not visible in the integrated distribution, we selected different center-of-mass angle intervals. Monte Carlo studies of the CLAS acceptance for this reaction showed that we could detect events over the entire angular range $\left(0^{\circ}-180^{\circ}\right)$, with some reduction of efficiency at forward angles $\left(\theta_{\bar{K}^{0}}^{C M}<30^{\circ}\right)$. No structures were found in the distribution when specific angular ranges were selected.

Since no signal was found, an upper limit for the $\Theta^{+}$production cross section in this reaction channel was extracted. The $n K^{+}$mass distribution was fit using the maximum likelihood procedure to the sum of a narrow Gaussian function and a $5^{\text {th }}$-order polynomial that parameterizes, respectively, the $\Theta^{+}$contribution and a smooth background. The resonance position was varied from 1520 to $1600 \mathrm{MeV}$ in $5 \mathrm{MeV}$ steps while the width was fixed at $2.5 \mathrm{MeV}$, a value derived by Monte Carlo simulation for a long-lived resonance and following the same analysis chain used to process the data. The measured yields were then used to evaluate an upper limit on the $\Theta^{+}$yield using the Feldman and Cousins approach [28]. The upper limit on the number of $\Theta^{+}$events at the $95 \%$ confidence level was compared with the number of observed $\Lambda^{*}(1520)$ 's shown in the upper panel of Fig. 2, which was estimated using a Breit-Wigner resonance shape fit. The ratio between the two yields is $\sim 220 / 100 \mathrm{k}=0.22 \%$. The upper limit on the yields was then transformed into an upper limit on the $\Theta^{+}$production cross section taking into account the luminosity of incident photons and target, the CLAS detection acceptance, the $\Theta^{+}$ branching ratio to $n K^{+}$of $50 \%$, and several models for the production mechanism. The upper panel in Fig. 4 shows the upper limit on the total cross section as a function of the $\Theta^{+}$mass obtained in the most conservative scenario (forward peaked center-of-mass production). An upper limit of $0.8 \mathrm{nb}$ was found for $\mathrm{M}=1540 \mathrm{MeV}$ including a systematic uncertainty of $20 \%$ estimated by comparing different analysis procedures. The process to extract the yield described above was repeated for each angular bin to derive the $95 \%$ CL upper limit on the $\Theta^{+}(1540)$ differential cross section. The result is shown in the lower panel of Fig. 4. The cross section upper limit remains within about 1-2 $\mathrm{nb}$ for most of the angular range and rises at forward angles due to the reduced CLAS acceptance.

Our upper limit on the cross section is in clear disagreement with the findings of Ref.[6] which reported a $\Theta^{+}$signal of 55 events at a mass of $1540 \mathrm{MeV}$ corresponding to the published total cross section of $300 \mathrm{nb}$. In order to better compare with that experiment, we repeated the analysis applying the same cuts reported in that paper: the photon energy was limited to $2.6 \mathrm{GeV}$, only events with a forward-emitted $\bar{K}^{0}$ $\left(\theta_{C M}^{\bar{K}^{0}}>60^{\circ}\right)$ were used and the hyperons were not cut. The resulting mass distribution is shown in the inset of Fig. 2: it remains smooth and structureless.

\section{CONCLUSIONS}

Spurred by an initial report by the LEPS Collaboration in 2003, many experimental collaborations reported possible evidence for a pentaquark state. The observed signals 

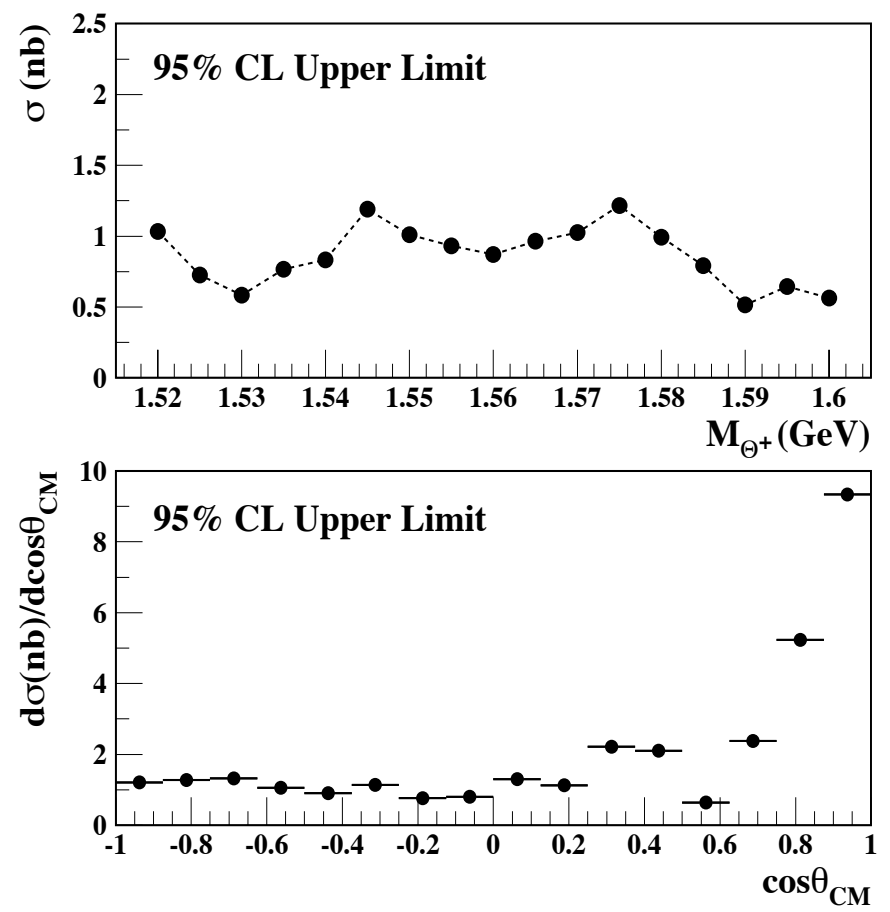

FIGURE 4. The $95 \%$ CL upper limit on the total cross section as a function of the $\Theta^{+}$mass (top) and on the differential cross section $d \sigma / d \cos \theta_{C M}^{\bar{K}^{0}}$ (bottom) for the reaction $\gamma p \rightarrow \bar{K}^{0} \Theta^{+}$for an assumed $\Theta^{+}$ mass of $1540 \mathrm{MeV}$. The dotted line in the top plot is to guide the eye.

suffered from low statistics and were challenged by null results reported last year by many other experiments in different kinematic regions and reactions. Our analysis of a dedicated high-statistics and high-resolution measurement conducted under similar kinematic conditions and with more than ten times the statistics of a previously reported positive result found that there is no evidence for a $\Theta^{+}$pentaquark in $\bar{K}_{0} K^{+} n$ reaction channel setting an upper limit of less than $1 \mathrm{nb}$ on the production cross section. Analysis of other reaction channels on the new JLab data are still underway and expected to release results in a short time.

\section{REFERENCES}

1. D. Diakonov, V. Petrov and M. Polyakov, Z. Phys. A 359, 305 (1997).

2. T. Nakano et al. (LEPS Collaboration), Phys. Rev. Lett. 91, 012002 (2003).

3. S. Stepanyan et al. (CLAS Collaboration), Phys. Rev. Lett. 91, 252001 (2003).

4. V. Kubarovsky et al. (CLAS Collaboration), Phys. Rev. Lett. 92, 032001 (2004).

5. V. V. Barmin et al. (DIANA Collaboration), Phys. Atom. Nucl. 66, 1715 (2003).

6. J. Barth et al. (SAPHIR Collaboration), Phys. Lett. B 572, 127 (2003).

7. A.E. Asratyan, A.G. Dolgolenko, and M.A. Kubantsev, Phys. Atom. Nucl. 67, 682 (2004). 
8. A. Airapetian et al. (HERMES Collaboration), Phys. Lett. B 585, 213 (2004).

9. M. Abdel-Bary et al. (COSY-TOF Collaboration), Phys. Lett. B 595, 127 (2004).

10. S. Chekanov et al. (ZEUS Collaboration), Phys. Lett. B 591, 7 (2004).

11. A. Aleev et al. (SVD Collaboration), Phys. At. Nucl. 68, 974 (2005) and hep-ex/0509033.

12. A. Aktas et al. (H1 Collaboration), Phys. Lett. B 588, 17 (2004).

13. S. Schael et al. (ALEPH Collaboration), Phys. Lett. B 599, 1 (2004).

14. B. Aubert et al. (BABAR Collaboration), arXiv:hep-ex/0408064.

15. K. Abe et al. (Belle Collaboration]), arXiv:hep-ex/0411005.

16. J. Z. Bai et al. (BES Collaboration), Phys. Rev. D 70, 012004 (2004).

17. I. V. Gorelov (CDF Collaboration), arXiv:hep-ex/0408025; D. O. Litvintsev (CDF Collaboration), Nucl. Phys. Proc. Suppl. 142, 374 (2005).

18. K. Stenson (FOCUS Collaboration), arXiv:hep-ex/0412021.

19. I. Abt et al. (HERA-B Collaboration), Phys. Rev. Lett. 93, 212003 (2004); K. T. Knopfle, M. Zavertyaev and T. Zivko (HERA-B Collaboration), J. Phys. G 30, S1363 (2004).

20. M. J. Longo et al. (HyperCP Collaboration), Phys. Rev. D 70, 111101 (2004).

21. J. Napolitano, J. Cummings and M. Witkowski, arXiv:hep-ex/0412031.

22. S. R. Armstrong, Nucl. Phys. Proc. Suppl. 142, 364 (2005).

23. C. Pinkenburg (PHENIX Collaboration), J. Phys. G 30, S1201 (2004).

24. Y. M. Antipov et al. (SPHINX Collaboration), Eur. Phys. J. A 21, 455 (2004).

25. M. I. Adamovich et al. (WA89 Collaboration), arXiv:hep-ex/0405042.

26. M. Ostrick, Prog. Part. Nucl. Phys. 55, 337 (2005).

27. B. Mecking et al.,Nucl. Instrum. and Meth. A503, 513 (2003).

28. G. J. Feldman and R. D. Cousins, Phys. Rev. D 573873 (1998). 